\title{
A NEW STRATEGY FOR PHASE UNWRAPPING IN INSAR TIME SERIES OVER AREAS WITH HIGH DEFORMATION RATE: CASE STUDY ON THE SOUTHERN TEHRAN SUBSIDENCE
}

\author{
P. Ajourlou ${ }^{1}$, S. Samiei Esfahany ${ }^{1, *}$, A. Safari ${ }^{1}$ \\ ${ }^{1}$ Department of Surveying and Geomatics Engineering,University of Tehran,Tehran,Iran; \\ (parviz.ajourlou, s.samieiesfahany, asafari)@ut.ac.ir
}

KEY WORDS: Phase Unwrapping, Subsidence, Radar Interferometry

\begin{abstract}
:
The primary step in all timeseries interferometric synthetic aperture radar (T-InSAR) algorithms is the phase unwrapping step to resolve the inherent cycle ambiguities of interferometric phases. In areas with a high spatio-temporal deformation gradient, phase unwrapping fails due to the aliasing problem, and so it can result in an underestimation of deformation signal. One way to handle this problem is to use the so called Small-Baseline Subset (SBAS) algorithms; in these algorithms, by using only small-baseline interferograms - hence interferograms with small deformation gradients - the chance of unwrapping error gets reduced. However, due to more number of the used interferograms, SBAS method is computationally more expensive and more time-consuming compared to algorithms that exploit Single-Master (SM) stacks. Moreover, the existence of sufficiently small temporal baseline interferograms is not guaranteed in all SAR stacks. In this paper, we propose a new method to take advantage of short temporal baseline interferograms but effectively using SM approach. We treat the phase unwrapping step as a Bayesian estimation problem while the prior information, required by the Bayesian estimator, is extracted from few short coherent interferograms that are unwrapped separately. Results from the proposed approach and a case study over the southwest of Tehran, with a high subsidence rate (reaching to $25 \mathrm{~cm} /$ year), demonstrates that utilizing the proposed method overcomes the aliasing problem and produces the results equal to the conventional SBAS results, while the proposed method is computationally much more efficient than SBAS.
\end{abstract}

\section{INTRUDUCTION}

During the last decades, time-series interferometric synthetic aperture radar (T-InSAR) has emerged as a powerful technique to measure various surface deformation phenomena of the earth. The primary step in all T-InSAR algorithms is the phase unwrapping step to resolve the inherent cycle ambiguities of interferometric phases. As InSAR phase observations are wrapped (modulo- $2 \pi$ ), the estimation of the unknown absolute phase from the wrapped observations is always required in order to obtain deformation signals. From an estimation point of view, the unwrapping problem is inherently undetermined, and hence non-unique. As a consequence, it is impossible to solve the unwrapping problem without any a-priori knowledge or assumption about the signal of interest. The most common assumption, traditionally used in InSAR, is that the difference between the phase of two adjacent pixels is not more than half a wave cycle. However, in areas with a high spatial-temporal gradient of signal components (e.g high gradient of deformation induce by an earthquake close to a fault or high gradient subsidence), this assumption may be violated. It is well known that, when the deformation gradient is large in time/space, it can cause temporal/spatial aliasing of the signal in the phase unwrapping step, and, consequently, creates the so-called unwrapping errors. These errors can result in an underestimation of the obtained deformation fields in areas with high spatio-temporal deformation gradient (Chen , Zebker, 2000). As the deformation gradient in short temporal baseline interferograms is relatively smaller, the probability of unwrapping errors would be reduced by using only small temporal baseline interferograms. Therefore, in the areas with a high deformation gradient, the application of

\footnotetext{
${ }^{*}$ Corresponding author
}

Small-Baseline Subset (SBAS) algorithms (Berardino et al., 2002) is advantageous compared to the methods exploiting single-master (SM) stacks (Ferretti et al., 2000, Hooper, 2006). However, the existence of sufficiently small temporal baseline interferograms is not guaranteed in all SAR stacks. Moreover, the main drawback of the SBAS methodology compared to the SM methods is that it is computationally more expensive and more time-consuming. This limitation is remarkable in large SAR stacks, which is common these days, e.g., with the Sentinel-1 data with more than hundreds of acquisitions over each area.

In this paper, we propose a new approach to simultaneously take advantage of short temporal baselines regarding the aliasing problem, while using effectively SM algorithms that are computationally more efficient. In the proposed approach, we use SM stacks of interferograms, but we treat the phase unwrapping step as a Bayesian estimation problem. The prior information, required by the Bayesian estimator, is extracted from few short temporal baseline interferograms that are unwrapped separately, and are averaged subsequently to provide an initial guess of the magnitude of the deformation field. Then this initial guess is used as the prior information in the Bayesian phase unwrapping of the SM interferograms. We tested the proposed method over the subsidence field of the southwestern Tehran whose subsidence rate is relatively high (reaching to $25 \mathrm{~cm} /$ year). We used the whole Sentinel-1 data archive over the area. The results confirm that the standard SM method underestimates the deformation rate significantly. Utilizing the new approach overcomes the aliasing problem and produces the results equal to the results of the conventional SBAS, while the proposed method is computationally much more efficient than SBAS. Note that the proposed mathematical 
framework, is flexible to digest prior information also from other sources than the InSAR stack, for example from previous studies or from auxiliary observations, such as GNSS and leveling data.

This paper is organized as follows. Section 2 recaps the theory of the phase unwrapping problem and introduces the theoretical framework of phase unwrapping algorithms. Section 3 describes, in details, the proposed method. Then in section 4 , the results of the case study over the southern Tehran is presented, followed by the conclusions and discussion in section 5 .

\section{BACKGROUND KNOWLEDGE ON PHASE UNWRAPPING}

The most crucial processing step in T-InSAR methodologies is the phase unwrapping. It is crucial because the problem is inherently ill-posed and non-unique. As a consequence, a-priori information or assumptions about the signals of interest should be introduced in order to solve the unwrapping problem. Some methods assume a pre-defined kinematic behavior in time, and so they solve the problem primarily in the time domain (i.e., 1D unwrapping) (Ferretti et al., 2000, van Leijen, 2014), while other methods assume a predefined spatial deformation behavior and solve the problem mainly in the spatial domain (Goldstein et al., 1988, Costantini, 1998, Chen, 2001). ${ }^{1}$

To limit the scope of this study, we mainly focus on T-InSAR algorithms that apply unwrapping in the space domain (i.e. 2D unwrapping), for example in (Hooper, 2006). In these algorithms, the main strategy is to unwrap every interferogram spatially by the minimum cost optimization (Chen, 2001), where cost functions are used to insert the required a-priori knowledge in the unwrapping problem. In the rest of this section, we first recap on the optimization methods for phase unwrapping, following by explanation of common strategies to construct the cost functions.

\section{Spatial Unwrapping by Minimum Cost Optimization}

The main objective of $2 \mathrm{D}$ phase unwrapping is to estimate unwrapped phases $\phi$ from wrapped phases $\phi^{w}$ given the constrain of congruency (i.e., the wrapped and unwrapped phases should differ only by integer numbers of cycles). Usually, instead of working with absolute phases, the phase differences (i.e., arcs) between adjacent pixels are used in the unwrapping formulation. That is the objective is to estimate unwrapped phase gradients $\Delta \phi$ from wrapped gradients $\Delta \phi^{w}$ given the constrain that the difference between the two should be equal to integer numbers of cycles. This estimation problem is formulated in a general form as an optimization problem (Chen, Zebker, 2001):

$$
\Delta \hat{\phi}=\operatorname{argmin}_{\Delta \phi} \sum_{k} g\left(\Delta \phi_{k}, \Delta \phi_{k}^{w}\right)
$$

where $k$ is the index of all the phase gradients in both range and azimuth direction, and $g\left(\Delta \phi, \Delta \phi^{w}\right)$ is the cost function. Early

\footnotetext{
${ }^{1}$ It should be noted that whatever method is used (1D or $2 \mathrm{D}$ ), the ambiguities (i.e. the unknown integer number of phase cycles) always, and in all unwrapping methods, are estimated for phase differences in time and space or, in other words, double difference phases. Hence unwrapping is essentially always performed in $3 \mathrm{D}$, in both space and time. From this perspective the popular terminologies such as "1D unwrapping" or "2D unwrapping" may be misleading.
}

formulations of the 2D unwrapping problem, use a function of the difference between $\Delta \phi$ and $\Delta \phi^{w}$ as the cost function and use $L^{p}$-norm minimization (Ghiglia , Romero, 1996):

$$
g\left(\Delta \phi_{k}, \Delta \phi_{k}^{w}\right)=w\left|\Delta \phi, \Delta \phi^{w}\right|^{p}
$$

With this formulation, the cost functions have the same shape that is determined by the constant $p$, where $w$ denotes the weight assign to each gradient. When $p=2$ the problem is the same as weighted least squares minimization. Different algorithms exist for this $L^{p}$-norm minimization with $p$ is equal to 0,1 , or 2 (Pritt, 1996, Fornaro et al., 1996, Costantini, 1998, Goldstein et al., 1988). The drawback of these class of cost functions is that they all are formulated purely based on mathematics and they do not have any physical basis, e.g., based on quality of interferograms or based on the physical behavior of the signal of interest (i.e., deformation mechanism). Alternatively, another formulation has been presented by (Chen, 2001, Chen, Zebker, 2001) that formulate the unwrapping problem in the Bayesian framework as a maximum a posteriori probability (MAP) estimation problem:

$$
\Delta \hat{\phi}=\underset{\Delta \phi}{\operatorname{argmax}} f_{\Delta \Phi}\left(\Delta \Phi \mid \Delta \Phi^{w}\right)
$$

where $\Delta \Phi$ and $\Delta \Phi^{w}$ are the vector of unwrapped and wrapped gradients, and $f_{\Delta \Phi}\left(\Delta \Phi \mid \Delta \Phi^{w}\right)$ is the conditional probability density function (PDF) of unwrapped phases condition at $\Delta \Phi^{w}$. It is shown that the above MAP estimator can be formulated (assuming no correlation between phase gradients) as

$$
\Delta \hat{\phi}=\underset{\Delta \phi}{\operatorname{argmax}} \prod_{k} f_{\Delta \underline{\phi}_{k}}\left(\Delta \phi_{k} \mid \Delta \phi_{k}^{w}\right)
$$

This maximization problem was fomulated as series of maximum a posteriori probability (MAP) estimation problems as (for the proof, see (Chen, 2001, Chen , Zebker, 2001)) :

$$
\Delta \hat{\phi}=\underset{\Delta \phi}{\operatorname{argmin}}\left\{\sum_{k}-\log \left(f_{\Delta \underline{\phi}_{k}}\left(\Delta \phi_{k}\right)\right)\right\} .
$$

This formulation has the same structure as equation (1) with cost functions as

$$
g\left(\Delta \phi_{k}, \Delta \phi_{k}^{w}\right)=-\log \left(f_{\Delta \underline{\phi}_{k}}\left(\Delta \phi_{k}\right)\right)
$$

Note that for unwrapping problems we need to also enforce the congruence in the optimization. In other words, with the cost function of equation (6), the $2 \mathrm{D}$ unwrapping problem is equivalent to the constraint optimization of equation (5) with the congruency constraint. A general example of PDF of unwrapped phase gradient $f_{\Delta \underline{\phi}_{k}}\left(\Delta \phi_{k}\right)$ has been visualized in figure 1. Note that, in order to enforcing congruence, we should work with a discrete probability mass function (PMF) instead of continuous PDF. In figure 1, the black dots gives the discrete probability mass function after enforcing congruence. This constraint optimization has been implemented in the well-known unwrapping software SNAPHU. The PDF of unwrapped phase gradient in this algorithm is assumed to be a normal distribution whose standard deviation described the precision of the observed gradient (and so it is dependent to the coherence of the area). The center/mean of the normal distribution is the initial guess/knowledge about the unwrapped phase gradients (Chen, Zebker, 2001, Hooper, 2009). In fact, the center of the distribution allows us to insert our a-priori 
knowledge/assumption about the deformation signal into the unwrapping solver.

Standard SNAPHU algorithm, use the normal distribution with zero mean, assuming that the phase difference between neighboring sample points in any dimension is generally less than half a phase cycle (the Nyquist criteria). However this assumption is not valid in areas with a high deformation gradient. To solve the problem, an alternative strategy has been introduced by (Hooper, 2006, Hooper, 2009). In this approach, before spatial unwrapping by optimization of equation (5), all the phase gradients are first unwrapped in time (assuming no phase jump larger than $\pi$ between two adjacent acquisitions), and then the unwrapped phases are used to assign the center of the normal PDF functions. In other words, the output of the $1 \mathrm{D}$ temporal unwrapping is used to guide the $2 \mathrm{D}$ unwrapping in space, and so this method sometimes is called 1D+2D unwrapping. This approach is mainly based on the assumption that: "the phase difference of a sample point with respect to a nearby sample point is likely to vary by less than half a cycle between acquisitions, because the contribution from spatially-correlated signals between points close in space is usually small" (Hooper, 2009). The main limitation of this assumption is in the areas with a high rate of deformation where this assumption does not hold. In order to handle this limitation, in the next section, we introduce a new strategy to construct the cost functions in areas with high deformation rate.

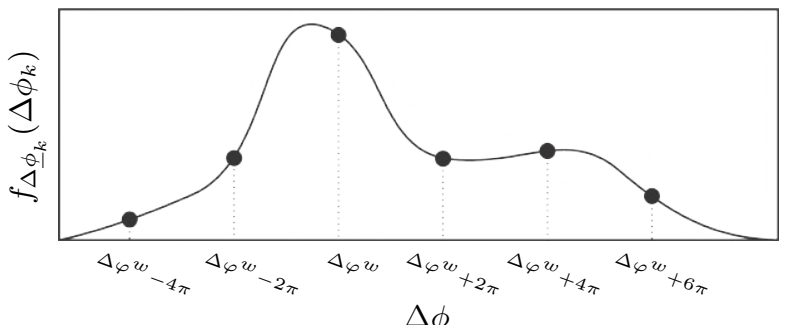

Figure 1. A general example of PDF of unwrapped phase gradient. The black dots gives the descrite probability mass function after enforcing congruence (not scaled).

\section{PROPOSED METHOD}

In the proposed approach, we use SM interferograms, and we use the Bayesian framework of the previous section for spatial unwrapping of interferograms. The main innovation is on how to make the cost function in in areas with high deformation rate. We propose to extract the prior information, required by the Bayesian method, from few (very) short temporal baseline interferograms that are unwrapped separately. In these coherent small-baseline interferograms, as the deformation gradients are small, the conventional unwrapping method (based on Nyquist criterion) work well and the chance of unwrapping errors are small. After unwrapping of the selected interferograms, to reduce systematic and orbital errors, we de-trend the unwrapped interferograms by fitting and subtracting a 2D plane.

Then, as unwrapped interferograms may have different masters, we resample them into a one super-master image that will be used as the master image of the SM stack. The unwrapped and resampled interferograms are combined subsequently to estimate an initial guess of the deformation rate field using the least squres estimation. From the estimated rates, the initial value for each phase gradient in every SM interferograms is calculated and assigned to the center of the cost functions

\begin{tabular}{ccccc}
\hline Dataset & time-span & RO & Pass & $\theta\left(^{\circ}\right)$ \\
\hline S1-A & Oct2014- May2018 & 28 & asc & $29-46$ \\
\hline \hline
\end{tabular}

Table 1. Overview of the used SAR stack. S1-A: Sentinel 1-A sensor, RO: relative orbit

required by the Bayesian estimator. In this way, we use the unwrapped phases of few good quality interferograms to guide the spatial unwrapping of all the SM interferograms. As our method use few small-baseline interferograms, we name it Semi-SBAS method. Figure 2 show an example of the distribution of temporal and perpendicular baselines of the radar images; dash lines represent SM combinations and solid lines represent the few small-baseline interferograms that are used for obtaining the initial deformation rates.

The main question, in this approach, is how to select the small-baseline interferograms and how many of them are required. In principle, even one coherent small-baseline interferogram is enough for extraction of the initial guess of deformation rate. But we recommend, to use small-baseline interferograms from different seasons and years to be able to capture seasonal variation and other short time deformation.

In the following section, we apply the proposed method over the subidence field of the southern Tehran, followed by the comparison of the results obtained from standard SM method.

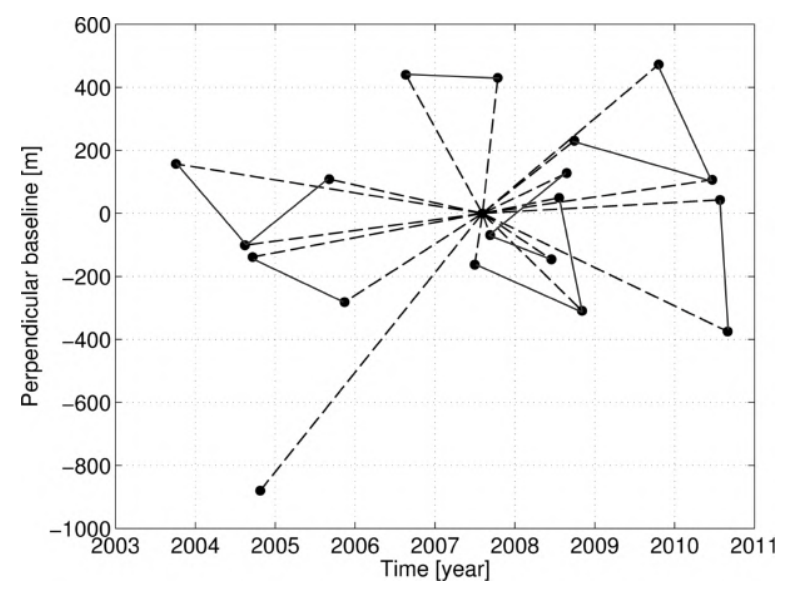

Figure 2. A baseline plot example for the proposed method: dash lines represent SM combinations and solid lines represent the few small-baseline interferograms that are used for obtaining the initial deformation rates.

\section{CASE STUDY}

We tested the proposed method over the subsidence field of the southwest Tehran whose subsidence rate is relatively high, reaching to $28 \mathrm{~cm} /$ year induce by water extraction (Motagh et al., 2008, Dehghani et al., 2013, Haghshenas Haghighi , Motagh, 2019). We used the whole Sentinel-1 ascending data archive (2014-2018) over the area including a stack of 72 Sentinel 1-A SAR images. The detail of the SAR stack is summarized in Table 1.

We have co-registered all slave images to a single master (16 Jul 2016 In ascending orbit), which was chosen to minimize the average value of perpendicular and temporal baselines. The distribution of both temporal and perpendicular baselines is illustrated in figure 3 . 


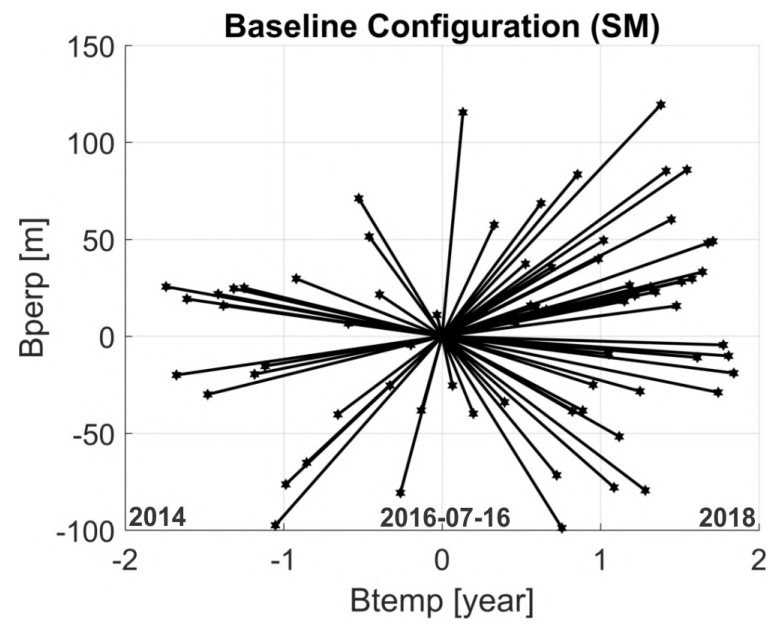

Figure 3. The baseline plot of SM interferograms

\section{Results from the Conventional SM method}

In this section, we have applied the conventional SM method for InSAR processing (STAMPS software). Figure 4 illustrates the result of subsidence in Tehran basin with conventional SM method. Results show the maximum of $14 \mathrm{~cm} /$ year subsidence in this area in satellite line of sight (LOS) direction. Assuming that deformation is mainly due to the vertical subsidence, as reported by (Haghshenas Haghighi , Motagh, 2019), the estimated maximum deformation is equal to $\sim 17$ $\mathrm{cm} /$ year vertical subsidence, which as much smaller than $\sim$ $25 \mathrm{~cm} /$ year reported in previous investigations (Motagh et al., 2008, Dehghani et al., 2013) and the recent results obtained by SBAS methodology (Haghshenas Haghighi, Motagh, 2019).

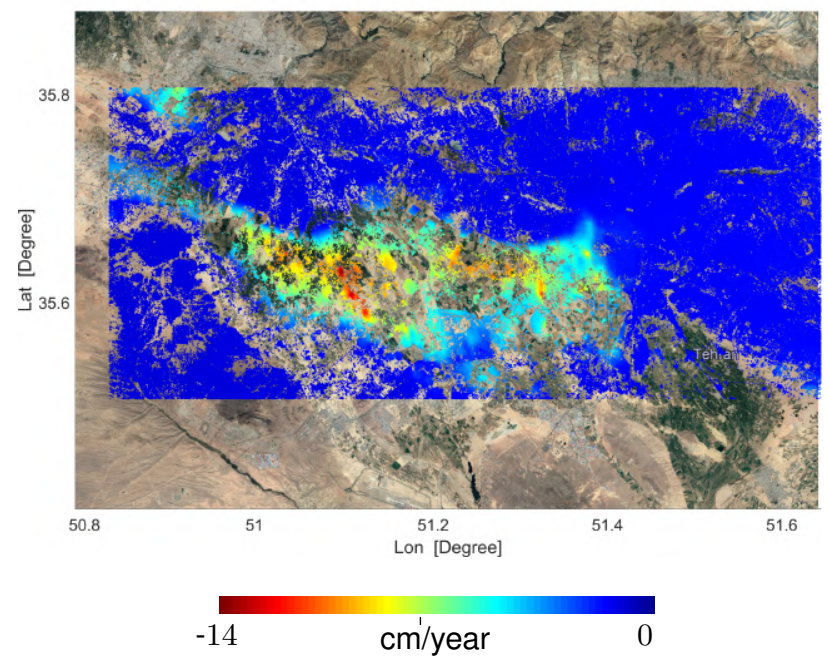

Figure 4. Tehran basin subsidence with conventional SM method in ascending orbit.

Also, the investigating a coherent interferogram with a five-month temporal baseline (figure.5) shows almost three fringes, equivalent to $8.4 \mathrm{~cm}$ LOS deformation in five months. This amount of deformation is equivalent to almost $20 \mathrm{~cm} /$ year LOS deformation or $24 \mathrm{~cm} /$ year vertical subsidence, which is much larger than the obtained results from the conventional SM method. We can conclude that, most likely, this underestimation is due to the aliasing problem in the phase unwrapping step of the STAMPS software.

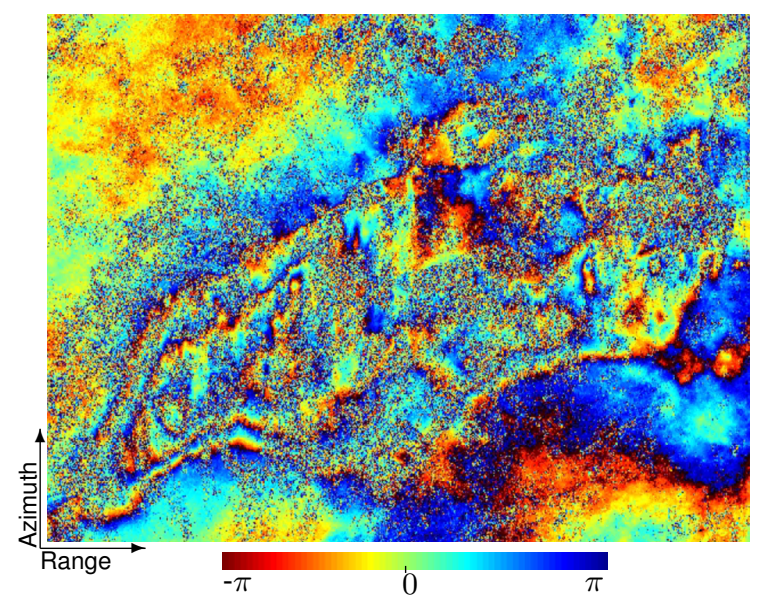

Figure 5. A five-months interferogram over Tehran basin, between February 2017 and July 2017. There are almost three fringes in the deformation area, equivalent to $8.4 \mathrm{~cm}$ LOS deformation in five months

\section{Results from the Proposed Method}

We selected few coherent interferograms with short baselines as visualized as red lines in figure. 6 . The a-priori deformation field obtained from these interferograms is shown in figure $7 \mathrm{~A}$. The gradient phases calculated from the a-priori deformations are used as the center of the cost functions of the SNAPHU algorithm in STAMPS software.

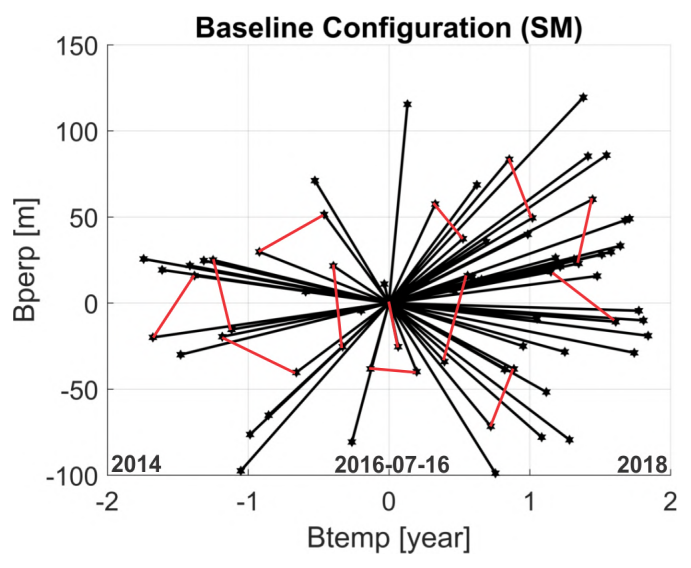

Figure 6. The distribution of both temporal and perpendicular baselines of radar images in the new method. Solid lines represents SM interferogram, and red

lines represents the selected SB interferogram for obtaining a-priori defromation field.

Figure $7 \mathrm{~B}$ shows the result of the proposed method, where the difference with the a-priori deformation has been depicted in figure $7 \mathrm{C}$. The results confirm that the standard SM method underestimates the deformation rate significantly. Utilizing the proposed semi-SBAS method overcomes the aliasing problem and produces the results equal to the results of the conventional SBAS (as reported by (Haghshenas Haghighi , Motagh, 2019)). Note the proposed method is computationally much more efficient than SBAS as it requires smaller number of interferograms. Results show the maximum of $17.5 \mathrm{~cm} /$ year 


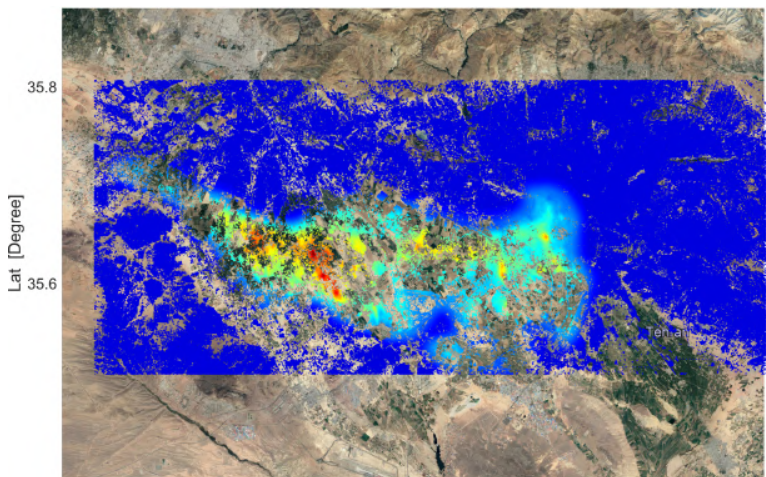

(A)

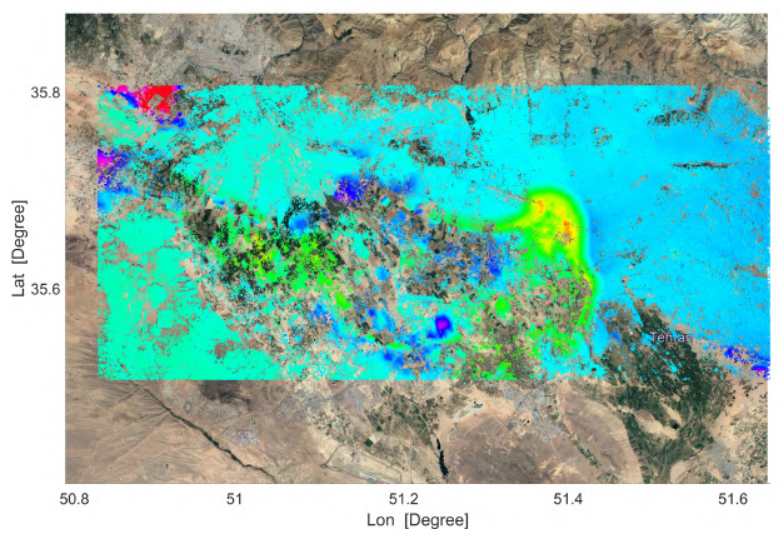

(C)

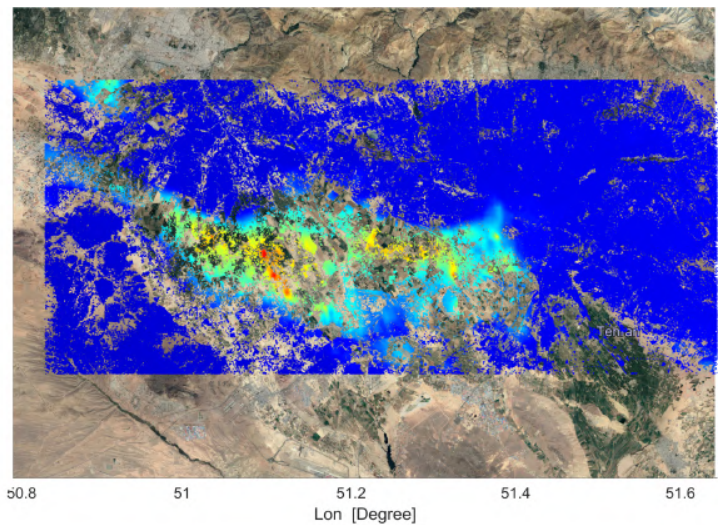

(B)

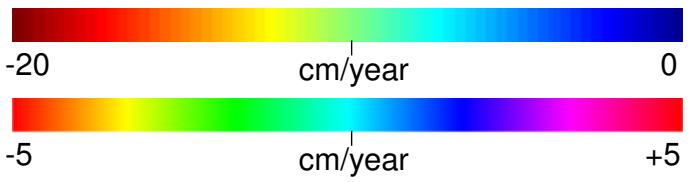

Figure 7. Tehran basin subsidence with Semi-SBAS method in ascending orbit. A) prior information. B) Tehran basin subsidence in ascending orbit. C) The difference between A and B.

deformation LOS direction, equivalent to $\sim 22 \mathrm{~cm} /$ year vertical deformation.

\section{CONCLUSION}

We proposed a new method to simultaneously take advantage of lower computational requirement of SM methods and the reliability of short temporal baseline interferograms regarding the aliasing problem in phase unwrapping. We modeled the phase unwrapping step as a Bayesian estimation problem while the prior information, required by the Bayesian estimator, is extracted from few short coherent interferograms that are unwrapped separately. Results from the proposed approach and a case study over the southwest of Tehran, with a high subsidence rate (reaching to $22 \mathrm{~cm} /$ year), demonstrates that utilizing the proposed method overcomes the aliasing problem and produces the results equal to the conventional SBAS results, while the proposed method is computationally much more efficient than SBAS.

\section{REFERENCES}

Berardino, Paolo, Fornaro, Gianfranco, Lanari, Riccardo, Sansosti, Eugenio, 2002. A New Algorithm for Surface Deformation Monitoring Based on Small Baseline Differential SAR Interferograms. IEEE Transactions on Geoscience and Remote Sensing, 40, 2375-2383.

Chen, Curtis W, 2001. Statistical-cost network-flow approaches to two-dimensional phase unwrapping for radar interferometry. $\mathrm{PhD}$ thesis, Stanford University.
Chen, Curtis W, Zebker, Howard A, 2000. Network approaches to two-dimensional phase unwrapping: intractability and two new algorithms. Journal of the Optical Society of America A., 17, 401-414. http://nova.stanford.edu/ curtis/research/netunw.pdf.

Chen, Curtis W., Zebker, Howard A., 2001. Two-dimensional phase unwrapping with use of statistical models for cost functions in nonlinear optimization. Journal of the Optical Society of America A, 18, 338-351. http://josaa.osa.org/abstract.cfm?URI=josaa-18-2-338.

Costantini, Mario, 1998. A Novel Phase Unwrapping Method Based on Network Programming. IEEE Transactions on Geoscience and Remote Sensing, 36, 813-821.

Dehghani, Maryam, Valadan Zoej, Mohammad Javad, Hooper, Andy, Hanssen, Ramon, Entezam Soltani, Iman, Saatchi, Sassan, 2013. Hybrid conventional and Persistent Scatterer SAR interferometry for land subsidence monitoring in the Tehran Basin, Iran. ISPRS Journal of Photogrammetry and Remote Sensing, 79, 157170.

Ferretti, Alessandro, Prati, Claudio, Rocca, Fabio, 2000. Nonlinear Subsidence Rate Estimation using Permanent Scatterers in Differential SAR Interferometry. IEEE Transactions on Geoscience and Remote Sensing, 38, 2202-2212.

Fornaro, G, Franceschetti, G, Lanari, R, 1996. A new efficient solution for DEM generation in interferometric SAR processing. European Conference on Synthetic Aperture Radar, Königswinter, Germany, 26-28 March 1996, 195-198.

Ghiglia, Dennis C, Romero, Louis A, 1996. Minimum $L^{p}$-norm two-dimensional phase unwrapping. Journal of the Optical Society of America A., 13, 1999-2013. 
Goldstein, Richard M, Zebker, Howard A, Werner, Charles L, 1988. Satellite radar interferometry: Two-dimensional phase unwrapping. Radio Science, 23, 713-720.

Haghshenas Haghighi, Mahmud, Motagh, Mahdi, 2019. Ground surface response to continuous compaction of aquifer system in Tehran, Iran: Results from a long-term multi-sensor InSAR analysis. Remote Sensing of Environment, 221, 534-550.

Hooper, Andrew, 2006. Persistent Scatterer Radar Interferometry for Crustal Deformation Studies and Modeling of Volcanic Deformation. PhD thesis, Stanford University.

Hooper, Andrew, 2009. A statistical-cost approach to unwrapping the phase of insar time series. Seventh International Workshop on ERS/Envisat SAR Interferometry, 'FRINGE09', Frascati, Italy, 2007, 7 pp.

Motagh, Mahdi, Walter, Thomas R., Sharifi, Mohammad A., Fielding, Eric, Schenk, Andreas, Anderssohn, Jan, Zschau, Jochen, 2008. Land subsidence in Iran caused by widespread water reservoir overexploitation. Geophysical Research Letters, 35, L16403.

Pritt, Mark D, 1996. Phase Unwrapping by Means of Multigrid Techniques for Interferometric SAR. IEEE Transactions on Geoscience and Remote Sensing, 34, 728-738.

van Leijen, Freek, 2014. Persistent Scatterer Interferometry based on geodetic estimation theory. PhD thesis, Delft University of Technology, Delft, the Netherlands. 